\title{
Prevalence of EBV Genotypes in Polish, Taiwanese and Arabic Healthy Students and Association Between Genotypes and 30-bp Deletion in the LMP-1 Gene Phylogenetic Analysis
}

\author{
DOROTA POLZ ${ }^{\star}$, ŁUKASZ PODSIADŁO, AGNIESZKA STEC \\ and MAŁGORZATA POLZ-DACEWICZ
}

Department of Virology, Medical University, Lublin, Poland

Submitted 10 May 2013, revised 1 June 2013, accepted 16 November 2013

Abstract

\begin{abstract}
The aim of this study was to compare the prevalence of EBV genotype and del-LMP-1 in saliva from Polish, Taiwanese and Arabic healthy students. The study group consisted of 56 healthy students; 24 of them Polish, 25 Taiwanese, and 7 Arabic. Typing was carried out using PCR with EBNA-2 primers. A detection of LMP-1 variants was also performed using PCR. EBV DNA was detected in 22 investigated samples (39.3\%). Type 1 of the virus was dominant in both Polish and Taiwanese group. Among 62.5\% Tai wanese with EBV 1 and $55.6 \%$ Polish detected EBV with 30-bp deletion in LMP-1 gene.
\end{abstract}

Ke y words: EBV, saliva, LMP-1

\section{Introduction}

Epstein-Barr virus belongs to the Herpesviridae family, Gammaherpesviridae subfamily, Lymphocryptovirus genus and is also known as human herpesvirus 4 (HHV-4). Herpesviruses have double-stranded DNA genome (Peh et al., 2003). EBV is an enveloped virus with an icosahedral capsids symmetry. The genome takes on a linear form in mature virions and a circular episomal form during the period of latency in the infected cells. Epstein-Barr virus express six nuclear proteins: EBNA (EBV nuclear antigens) which include EBNA-1, EBNA-2, EBNA-3A, EBNA-3B, EBNA-3C and EBNA-LP (leader protein), next are three latent membrane proteins LMP-1, LMP-2A, LMP-2B and two untranslated RNAs termed EBV-encoded RNA (EBER-1 and EBER-2). LMP-1 is a transmembrane protein, it is a major oncogenic protein of EpsteinBarr virus and EBV strain are categorized due to nucleotide base-pair (bp) changes in the LMP-1 gene. Del-LMP-1 (variant with deletion) has a greater tumorigenic potential thus being responsible for an increased risk of nasopharyngeal carcinoma (NPC), and is less immunogenic (Perera et al., 2010). This is one of the most common viruses in humans (Alipov et al., 2005). EBV was the first human virus to which oncogenic potential was attributed, and is classified as a group 1 carcinogen by the World Health Organization's International Agency for Research on Cancer (IARC working group 1997). EBV is correlated with nasopharyngeal and gastric carcinoma, squamous cell carcinoma, Hodgkin's lymphoma, and Burkitt's lymphoma (Slots 2005, Slots et al., 2006, Peh etal., 2003, Chen 2011, Tiwawech 2008, Perera 2010). Nasopharyngeal carcinoma is the most common head and neck cancer in southwestern China and Taiwan (Lin et al., 2001). This virus has the ability to replicate and enter a latency phase in B cells, which can lead to a development of viral types of cancer (Chen 2011). So far, two types of EBV (EBV-1 and EBV-2) have been distinguished, with the significant differences in the EBNA-2, EBNA-3A, EBNA-3B and EBNA-3C gene sequences (Peh et al., 2003, Correra et al., 2007, Tiwawech et al., 2008). Type 1 is found primarily in Europe, North and South America and Asia, while type 2 is mainly dominant in Africa(Peh et al., 2003). EBNA-2 protein plays a key role in initiation of carcinogenesis by disrupting mitotic checkpoints and causing chromosomal instability (Pan et al., 2009). EBV is an etiological agent of infectious mononucleosis also known as the kissing disease', because the virus spreads through direct mouth-to-mouth contact with saliva being the main

\footnotetext{
* Corresponding author: D. Polz, Department of Virology, Medical University, Lublin, Poland; e-mail: dorota.polz@umlub.pl
} 
vehicle for EBV transmission from human to human. Current investigations suggest that EBV is correlated with many diseases localized in the oral cavity such as gingivitis, periodontitis, pulpitis, periapical inflammations and periodontal abscesses (Slots 2005, Slots et al., 2006, Grinde and Olsen 2010).

\section{Experimental}

\section{Material and Methods}

Sample collection. The investigated material was saliva. Samples were obtained from healthy students of the Medical University of Lublin, Poland. The study group consisted of 56 healthy volunteers: 24 Polish, 25 Taiwanese, and 7 Arabic students. In the study group there were 26 female and 30 male in 18-37 years of age (22.2 average). Saliva samples were collected anonymously, and all volunteers were healthy; there was also an a survey containing clinical about general and infectious diseases data attached. The research received approval from the Ethics Committee number KE-0254/150/2010.

DNA extraction. DNA from $200 \mu \mathrm{l}$ of saliva samples was extracted using QIAamp DNA Mini Kit (Qiagen, Germany) according to the manufacturer's instructions.

PCR. All PCR reactions were carried out in final volume of $25 \mu \mathrm{l}$ using HotStartTaq DNA Polymerase (Qiagen, Germany). Concentrations of PCR reaction components were prepared as follows: $2.0 \mathrm{mM} \mathrm{MgCl}_{2}$, $0.2 \mathrm{mM}$ dNTPs, $0.5 \mu \mathrm{M}$ each forward and reverse primers and $0.5 \mathrm{U}$ of HotStart Taq polymerase. During each run samples were tested together with one negative and positive control.

Amplification of EBNA-2 gene. The nested PCR was carried out for amplification of EBNA-2. The sequence of primers used for PCR was as follows: outer pair 5'-TTT CAC CAA TAC ATG ACC C-3', 5'-TGG CAA AGT GCT GAG AGC AA-3' and inner pair 5'-CAA TAC ATG AAC CRG AGT CC-3', 5'-AAG TGC TGA GAG CAA GGC MC-3', $(\mathrm{R}=\mathrm{A} / \mathrm{G}, \mathrm{M}=\mathrm{A} / \mathrm{C})$. $2 \mu$ of extracted DNA was subjected to the PCR mixture with the concentration as described above. The first-round amplification consisted of activation of polymerase $95^{\circ} \mathrm{C}$ for 15 minutes, 35 cycles of $94^{\circ} \mathrm{C}$ for 1 minute, $55^{\circ} \mathrm{C}$ for 1 minute, $72^{\circ} \mathrm{C}$ for 2 minutes and the final extension at $72^{\circ} \mathrm{C}$ for 5 minutes. The secondround amplification was performed with $1 \mu$ of first round PCR product in 30 cycles with an annealing temperature at $60^{\circ} \mathrm{C}$. The amplicons $368 \mathrm{bp}, 473 \mathrm{bp}$ in length (depending on the EBV type EBV-1 and EBV-2, respectively) were separated on $2 \%$ agarose gel and purified using Gel-Out kit (A\&A Biotechnology, Poland) for further analysis. Purified PCR products were send to Genomed Warsaw, a company dealing with the field of genomics.

Amplification of LMP-1 gene. Primers used for the amplification were 5'-AGCGACTCTGCTGGAAATGAT-3' and 5'-TGATTAGCTAAGGCATTCCCA-3'. The reaction mixture containing $3 \mu$ of extracted DNA was amplified under following conditions: $95^{\circ} \mathrm{C}$ for 15 minutes of initial hot start activation, then 40 cycles of $94^{\circ} \mathrm{C}$ for 1 minute, $57^{\circ} \mathrm{C}$ for 1 minute, $72^{\circ} \mathrm{C}$ for 1 minute with the final extension at $72^{\circ} \mathrm{C}$ for $10 \mathrm{~min}$ utes. The PCR products were subjected to $3 \%$ agarose gel and LMP-1 variants (316 bp - wild type or type with 30-bp deletion) were analyzed.

Phylogenesis of EBV (phylogenetic analysis). The results of sequencing were analyzed using the following computer programs: Chromas Lite 2.0.0.0, ClustalX 2.1.0.0, GeneDoc 2.7.0.0 and BioEdit 7.0.9.1. Typing was performed using the BLAST algorithm (basic Local Alignment Search Tool; http://blast.ncbi.nlm.gov/Blast. cgi). EBNA-2 gene sequence were used in order to construct phylogenetic tree. Phylogeny was based on the maximum likelihood method (ML). This method requires the use of computer programs such as PAUP 4.0, ModelTest 3.7, PhyML 2.4.4 and MEGA 4.1. In order to choose the appropriate model of molecular evolution hLRTs (hierarchical Likelihood-Ratio Test) and AIC (Akaike Information Criterion) test were used. The reference strains sequences used in the study was taken from the public database GenBank (http://www. ncbi.nlm.nih.gov/genbank/).

Statistical analysis. PQ Stat 1.4.6. program was used for the statistical analysis, and the relationship between investigated parameters was verified by means of V-Cramer test. Statistical significance was defined as $\mathrm{P}<0.05$.

\section{Results}

EBV DNA was detected in 39.3\% (22) investigated samples, EBV type 1 was detected in 18 cases $(81.8 \%)$ and EBV type 2 was detected in 4 cases (18.2\%). The findings revealed that more Taiwanese students was EBV-positive compared to Polish and Arabic students (44\%, 37.5\% and $28.6 \%$ respectively). Type 1 of the virus was dominant in both Polish and Taiwanese group (100\% and $72.7 \%$ respectively). The phylogenetic tree and percentage similarity of EBNA-2 gene sequences are shown in Figure 1 and in Table I. In the group of Arabic students EBV was detected in 2 cases out of seven respondents, amounting to $26.8 \%$. The low number of EBV-positive representatives enables an interpretation of this result (1 case of EBV1 and EBV2 was detected).

Among 54.5\% Taiwanese and 55.6\% Polish students EBV with 30-bp deletion in LMP-1 gene was detected. 
Table I

Percentage similarity of EBNA-2 gene sequences.

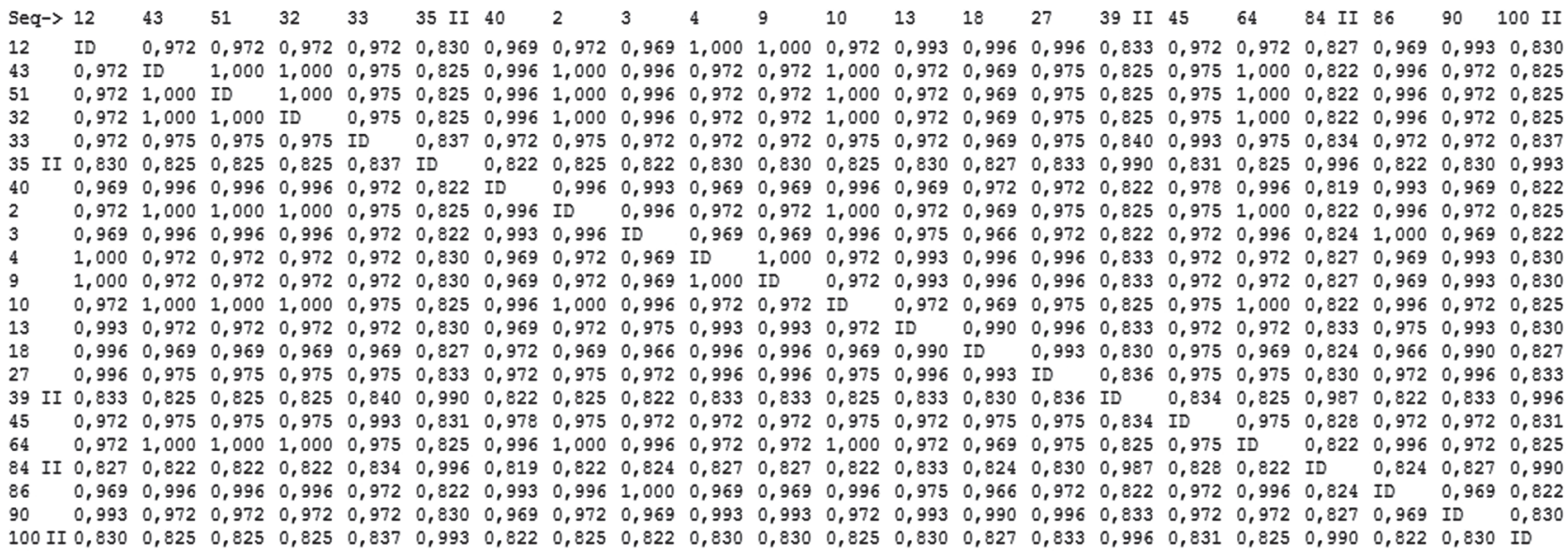

Among Taiwanese students with EBV 1 group deletion in LMP-1 gene was detected in 62.5\% (5 samples), so deletion in EBV 1 was more often detected among Taiwanese than among Polish students.

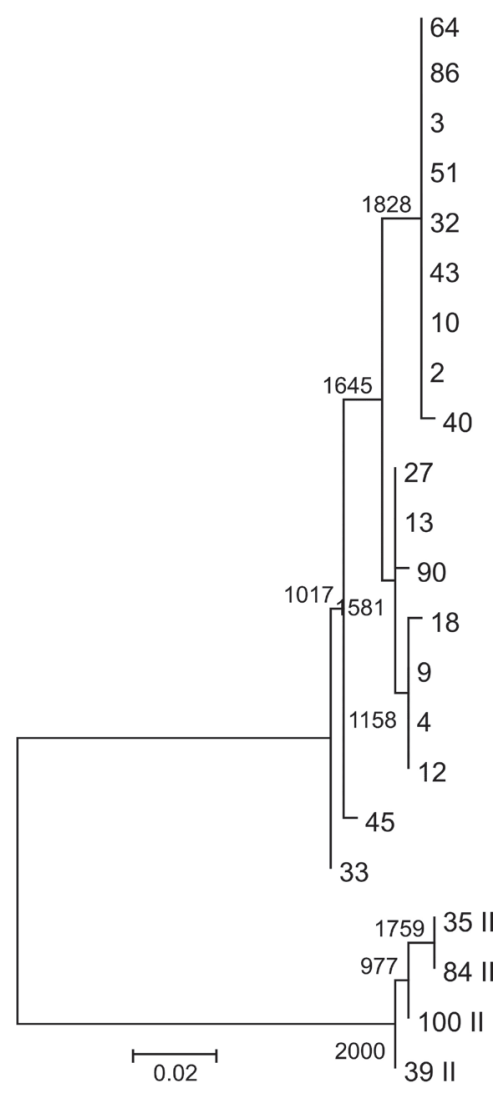

Fig. 1. Phylogenetic tree of EBV constructed based on the maximum likelihood method (ML).

\section{Discussion}

Saliva is a very interesting diagnostic material, sometimes described as the "mirror of the body". The undeniable benefit of this material is the way of obtaining samples; non-invasive nor stressful for the patient (especially when compared to collecting blood samples), the risk of needle stick is eliminated and at the same the risk of infection reduced. What is more, if blood is not visible in the saliva, the sample is not considered a class II biohazard (according to the US Centre for Disease Control) which also provides safety benefits for researchers. It is a painless method, which is of particular importance especially in the diagnosis of children. In addition, because of the way of collecting samples, it is inexpensive and the medical personnel does not require specialized training. Also the method of storing and preparing the material for further research is less complicated than in the case of serum (blood) (Shirtcliff et al., 2001). Saliva is used in the diagnosis of hormonal disorders (determination of cortisol concentration), it is considered to be good material for detecting oral cancer markers and monitoring the course of disease: the main biomarker is the tumour-suppressor protein TP53. Mutations in the p53 gene are considered to be the most common changes found in cancer, including squamous cell cancer of the oral cavity. Another cancer marker detected in saliva is CA125, used to diagnose ovarian cancer. Saliva as a diagnostic material has its application in virology: it is possible to detect anti-HIV IgM antibodies, using techniques of molecular biology (PCR) it is also possible to detect HSV, EBV, measles, and mumps (Farnaud et al., 2010).

EBV infection is transmitted from host to host via saliva, and the virus passes through the oropharyngeal epithelium to B lymphocytes (Thompson and Kurzrock 2004).

The Epstein-Barr virus belongs to a group of oncogenic viruses. The spectrum of diseases associated with being infected with this virus is very broad and the virus itself is common in the population. In our studies, the EBV DNA was detected in 39.3\% of the students. Type 1 was detected in all of the Polish students (100\%) while in the Taiwanese the detection of type 1 amounted to 
72.7\%. 30-bp deletion in the LMP-1 gene was detected in $54.5 \%$ of Taiwanese and $55.6 \%$ Polish students.

The results of our studies are consistent with the experiences of other authors e.g. higher incidence of EBV-1 in the healthy population is also confirmed by Correa et al. (2007).

LMP-1 EBV is a viral oncoprotein, which has an in vitro confirmation of the ability to transform cells and of inducing tumor growth (in vivo), it can disturb the growth and maturation of human keratinocytes and induce the expression of epidermal growth factor receptor connected to the nasopharyngeal carcinoma (NPC), and is probably associated with the tumor being more aggressive. Some researchers (Tiwawech et al., 2008 and Peh et al., 2003) report that the variant with deletion (del-LMP-1) is connected to changes which induce the protein's oncogenic properties, Sandvej etal. (1994) combine the EBV-2 and del-LPM-1, suggesting the genotype transforming ability. There is little information on prevalence of del-LMP-1 in healthy individuals. In Brazil and Mexico this deletion was detected in 59\% (Chen et al., 1996, Dirnhofer et al., 1999), among healthy Italian blood donors in 44\% (Dolcetti et al., 1997), in Argentina only in 7.4\% (Correa et al., 2004). Tiwawech et al. (2008) present studies where EBV-1 is dominant (86.5-96\%) in nasopharyngeal carcinoma (NPC) among Asians, and EBV-2 is less frequently detected (4-13\%). They also confirm a strong correlation between a virus with deletion in LMP-1 and NPC in comparison to the wild-type gene (without deletion) and NPC. An increasingly interesting and still valid is the question why EBV causes NPC only in some populations and maybe the answer lies within the genetic differences between the strains. Tiwawech et al. (2008) indicate that type $1(\mathrm{~A})$ of the virus has a greater potential of transforming B-lymphocytes than type II (B).

The result of studies of Tse et al. (2009) and Bei et al. (2010) (genome-wild association study) indicate that the increased incidence of NPC among the Taiwanese and eastern China population are an outcome of genetic differences in the human leukocyte antigen (HLA) and multiple loci (HLA-A, HLA-F, GABBR1) within chromosome 6 p 21.3 is associated with this type of cancer.

Also Perera et al. (2010) indicate that the genetic differences between populations may influence the degree of risk of NPC occurrence, they provide the example of HLA-A11 restricted cytotoxic T-lymphocyte response is directed toward a specific epitope of the EBNA-4 EBV protein. They also confirm the reports on the influence of genetic alterations in virus strains, which determine the active infections in humans. Available literature does not provide studies comparing nationalities studied by us, it is worth to underline however that the study was conducted among healthy young people. Perhaps this is related to the significant differences in the incidence of this cancer. Significant differences in NPC incidence depending on the geographical location were observed; in south-east Asia (an area covering southern China, Hong Kong, Taiwan), the annual incidence rate is about 25 times higher than in the western world (Tse et al., 2009). It is believed that NPC in South East Asia is endemic, while the annual incidence rate for the rest of the world amounts to less than 1/100 000 (Ruan et al., 2013). The incidence rate of NPC in the Guandong province (China) is 30.94/100 000 for men and 13/100 000 for women, in Taiwan it is estimated at $6 / 100000$, being eighth among cancers leading to death. Mortality is 4/100 000 and mainly concerns middle aged patients (Lin et al., 2001, Ruan et al., 2013). Hong Kong recorded some of the highest rates of incidence: 4.8/100 000 for women and 15.0/100 000 for men (Pow et al., 2011). In Poland, this type of cancer is rare among both men and women. In 2010, the absolute number of men affected with nasopharyngeal carcinoma was 119 , crude rate 0.6 , standardized rate 0.4 , percentage 0.2 . and is in place 49 in sequence number with regards to the location of particular organs- In the same years there were 50 cases of women suffering from the nasopharyngeal carcinoma, crude rate 0.3 , standardized rate 0.2 , percentage 0.1 , and is 67 in sequence number. NPC is so uncommon in Poland that there are no available data apart from those provided above (Wojciechowska et al., 2010).

EBV DNA is detected in the majority of cases of NPC among Asians and about 75\% of case among Caucasians (Pow et al., 2011).

Conclusions. As with other cancers, early detection is most significant for the prognosis. If the cancer is detected at an early stage, the patient's chances of survival increase. In case of cancers with a viral pathogenesis it is worth to consider the possibility of virological testing. Although in the Polish population NPC is rare, nevertheless it is recorded and one should remember about the correlation NPC/EBV DNA. What is more, in the age of globalization, the number of patients from the most remote parts of the world is likely to increase, and thus the number of rarely recorded diseases may also increase.

In conclusion, the knowledge on EBV prevalence in healthy population of Polish students will be useful in analyzing the role of EBV and regional factors in the pathogenesis of EBV-associated cancers.

\section{Literature}

Alipov G., T. Nakayama, M. Nakashima, C.Y. Wen, D. Niino, H. Kondo, Y. Pruglo and I. Sekine. 2005. Epstein-Barr virus-associated gastric carcinoma in Kazakhstan. World J. Gastroenterol. 11: 27-30. 
Bei J.X., Y. Li, W.H. Jia, B.J. Feng, G. Zhou, L.Z. Chen, O.S. Feng, HQ Low, H. Zhang, F. He and others. 2010. A genome-wide association study of nasopharyngeal carcinoma identifies three new susceptibility loci. Nat. Genet. 42: 599-603.

Chen W.G., Y.Y. Chen, M.M. Bacchi, C.E. Bacchi CE., M. Alvarenga and L.M. Weiss. 1996. Genotyping of Epstein-Barr virus in Brazilian Burkitt's lymphoma and reactive lymphoid tissue. Type A with a high prevalence of deletions within the latent membrane protein gene. Am. J. Pathol. 148: 17-23.

Chen M.R. 2011. Epstein-Barr virus, the immune system, and associated diseases. Front Microbiol. 2: 5.

Correa R.M., M.D. Fellner, L.V. Alonio, K. Durand, A.R. Teyssié, and M.A. Picconi. 2004. Epstein-Barr virus (EBV) in healthy carriers: Distribution of genotypes and $30 \mathrm{bp}$ deletion in latent membrane protein-1 (LMP-1) oncogene. J. Med. Virol. 73: 583-588.

Correa R.M., M.D. Fellner, K. Durand, L. Redini, V. Alonio, C. Yampolsky, A. Colobraro, G. Sevlever, A. Teyssié and others. 2007. Epstein Barr virus genotypes and LMP-1 variants in HIVinfected patients. J. Med. Virol. 79: 401-407.

Dirnhofer S., Angeles-Angeles A., Ortiz-Hidalgo C., Reyes E., Gredler E., J. Krugmann, F. Fend and L. Quintanilla-Martinez. 1999. High prevalence of a 30-base pair deletion in the Epstein-Barr virus (EBV) latent membrane protein 1 gene and of strain type $B$ EBV in Mexican classical Hodgkin's disease and reactive lymphoid tissue. Hum. Pathol. 30: 781-787.

Dolcetti R., P. Zancai, V. De Re, A. Gloghini, B. Bigoni, B. Pivetta, S. De Vita, A. Carbone and M. Boiocchi. 1997. Epstein-Barr virus strains with latent membrane protein-1 deletions: prevalence in the Italian population and high association with human immunodeficiency virus-related Hodgkin's disease. Blood. 89: 1723-1731.

Farnaud S.J., O. Kosti, S.J. Getting and D. Renshaw. 2010. Saliva: Physiology and Diagnostic Potential in Health and Disease. Scientific World Journal 10: 434-456.

Grinde B. and I. Olsen. 2010. The role of viruses in oral disease. J. Oral. Microbiol. 12: 2.

Lin SY., N.M. Tsang, S.C. Kao, Y.L. Hsieh, Y.P. Chen, C.S. Ksai, T.T. Kuo, S.P. Hao, I.H. Chen and J.H. Hong. 2001. Presence of Epstein-Barr virus latent membrane protein 1 gene in the nasopharyngeal swabs from patients with nasopharyngeal carcinoma. Head Neck. 23: 194-200.

Pan S.H., C.C. Tai, C.S. Lin, W.B. Hsu, S.F. Chou, C.C. Lai, J.Y. Chen, H.F. Tien, F.Y. Lee and W.B. Wang. 2009. Epstein-Barr virus nuclear antigen 2 disrupts mitotic checkpoint and causes chromosomal instability. Carcinogenesis 30: 366-375.
Peh S.C., L.H. Kim, K.S. Mun, E.L. Tan, C.K. Sam and S. Poppema. 2003. Epstein-Barr virus (EBV) subtypes and variants in malignant tissue from Malaysian patients. J. Clin. Exp. Hematopathol. 43: 61-69.

Perera RA., L.P. Samaranayake and C.S. Tsang. 2010. Shedding dynamics of Epstein-Barr virus: A type 1 carcinogen. Arch. Oral. Biol. 55: 639-647.

Pow E.H., M.Y. Law, P.C. Tsang, R.A. Perera and D.L. Kwong. 2011 Salivary Epstein-Barr virus DNA level in patients with nasopharyngeal carcinoma following radiotherapy. Oral. Oncol. 9: 879-882. Proceedings of the IARC working group on the evaluation of carcinogenic risks to tumors. Epstein-Barr virus and Kaposi's sarcoma Herpesvirus/Human herpesvirus 8. Lyon, France, 17-24 June 1997. IARC Monogr Eval Cacinog Risks Hum. 1997; 70: 1-492

Shirtcliff E.A., D.A. Granger, E. Schwartz and M.J. Curran. 2001. Use of salivary biomarkers in biobehavioral research: cotton-based sample collection methods can interfere with salivary immunoassay results. Psychoneuroendocrinology 26: 165-73

Slots J. 2005. Herpesviruses in periodontal diseases. Periodontol 2000. 38: 33-62.

Slots J., I. Saygun, M. Sabeti and A. Kubar. 2006. Epstein-Barr virus in oral diseases. J. Periodontal. Res. 41: 235-244.

Ruan H.L., H.D. Qin, Y.Y. Shugart, J.X. Bei, F.T. Luo, Y.X. Zeng and W.H. Jia. 2013. Developing genetic epidemiological models to predict risk for nasopharyngeal carcinoma in high-risk population of China. PLoS One. 82:e56128. doi: 10.1371/journal.pone.0056128 Sandvej K., S.C. Peh, B.S. Andresen and G. Pallesen. 1994. Identification of potential host spots in the carboxy-terminal part of Epstein-Barr virus (EBV) BNLF-1 gene in both malignant and benign EBV-associated diseases; High frequency of a 39-bp deletion in Malaysian and Danish peripheral T-cell lymphomas. Blood. 84: 4053-4060

Thompson M.P. and R. Kurzrock. 2004. Epstein-Barr virus and cancer. Clin. Cancer. Res. 10: 803-821

Tiwawech D., P. Srivatanakul, A. Karalak and T. Ishida. 2008. Association between EBNA2 and LMP1 subtypes of Epstein-Barr virus and nasopharyngeal carcinoma in Thais. J. Clin. Virol. 42: 1-6.

Tse KP., W.H. Su, K.P. Chang, N.M. Tsang, C.J. Yu, P. Tang, L.C. See, C. Hsueh, M.L. Yang, S.P. Hao and others. 2009. Genome-wide association study reveals multiple nasopharyngeal carcinoma-associated loci within the HLA region at chromosome 6p21.3. Am. J. Hum. Genet. 852: 194-203

Wojciechowska U., J. Didkowska and W. Zatoński. 2012. Cancer in Poland in 2010, http://www.onkologia.org.pl/doc/Nowotw2010.pdf 
